\title{
When to Use Different Inferential Methods for Power Analysis and Data Analysis for Between-Subjects Mediation
}

\author{
Jessica L. Fossum ${ }^{1}$ and Amanda K. Montoya ${ }^{2}$ \\ ${ }^{1}$ Seattle Pacific University \\ ${ }^{2}$ University of California - Los Angeles
}

\begin{abstract}
Author Note
Jessica L. Fossum (D) https://orcid.org/0000-0002-4907-0160

Correspondence concerning this article should be addressed to Jessica L. Fossum, Department of Psychology, Seattle Pacific University, 3307 Third Ave. W., Suite 107, Seatle, WA 98119. E-mail: fossumj@spu.edu
\end{abstract}




\begin{abstract}
Several options exist for conducting inference on indirect effects in mediation analysis. While methods which use bootstrapping are the preferred inferential approach for testing mediation, they are time consuming when the test must be performed many times for a power analysis. Alternatives which are more computationally efficient are not as robust, meaning accuracy of the inferences from these methods are more affected by nonnormal and heteroskedastic data (Biesanz et al., 2010). Previous research showed that different sample sizes are needed to achieve the same amount of statistical power for different inferential approaches with data that meets all the statistical assumptions of linear regression (Fritz \& MacKinnon, 2007). By contrast, we explore how similar power estimates are at the same sample size, including when assumptions are violated. We compare the power estimates from six inferential methods for between-subjects mediation using a Monte Carlo simulation study. This study varies the path coefficients, inferential methods for the indirect effect, and degree to which assumptions are met. We found that when the assumptions of linear regression are met, three inferential methods consistently perform similarly: the joint significance test, the Monte Carlo confidence interval, and the percentile bootstrap confidence interval. When the assumptions were violated, the non-bootstrapping methods tended to have vastly different power estimates compared to the bootstrapping methods. Based on these results, we recommend using the more computationally efficient joint significance test for power analysis only when no assumption violations are hypothesized a priori. We also recommend the joint significance test to pick an optimal starting sample size value for power analysis using the percentile bootstrap confidence interval when assumption violations are suspected.
\end{abstract}

Keywords: mediation analysis, indirect effect, power analysis, joint significance test, percentile bootstrap confidence interval, Monte Carlo simulation 


\section{When to Use Different Inferential Methods for Power Analysis and Data Analysis for Between-Subjects Mediation}

Mediation analysis examines how much a predictor variable affects an outcome variable through a mediator variable, described as the indirect effect (MacKinnon et al., 2004). According to a WebofScience search, over 10,000 published articles across many disciplines used the technique in 2021 alone. Within that, over 3,000 were from psychology. To effectively use these models, researchers can conduct a power analysis to determine an appropriate sample size given the size of effects they are expecting (Cohen, 1992). Ensuring adequate power prevents valuable research resources from being wasted by either collecting an unnecessarily large sample when fewer would be sufficient, or collecting too small of a sample and missing an effect that actually exists.

One way to select a sample size is through power analysis. A sample size can be calculated by setting a desired power level and false positive type 1 error rate $(\alpha$-level), along with setting reasonable estimates for the population effect sizes (for further information on the challenges of estimating reasonable effect sizes, see Anderson et al., 2017). Power analysis for mediation also requires the researcher to first choose an inferential method. Several methods exist to make inferences about the indirect effect, each varying in statistical power (Hayes \& Scharkow, 2013; Yzerbyt et al., 2018). These inferential methods also vary in computational efficiency, with the resampling methods that rely on bootstrapping being more time consuming (Shrout \& Bolger, 2002). This is particularly problematic for mediation, because power analyses using bootstrapping require computer simulations where the analysis is repeated many times. However, a major benefit to these methods are that they provide inferences that are robust to violations of normality and homoskedasticity (Biesanz et al., 2010). The purpose of this research is to evaluate whether power estimates among the methods are similar enough for researchers to use a more computationally efficient inferential method for power analysis, then a more computationally intensive test for data analysis, with added benefits such as robustness to 
assumption violations and production of a confidence interval (CI) that justify the computational time.

Fritz and MacKinnon (2007) explored differences in sample sizes requirements among methods of testing the indirect effect and provided sample size guidelines for commonly observed coefficients for each inferential method of the indirect effect at the same level of power. Their analyses and subsequent recommendations focused entirely on data that met statistical assumptions of linear regression, including normality and homoskedasticity of residuals. Because Fritz and MacKinnon (2007) found that the methods required different sample sizes to reach the same level of power, a researcher might conclude that they must always use the same inferential method for their power analysis as their data analysis. The current research questions this approach, since it can necessitate using a method that takes much longer for power analysis due to benefits for data analysis which are not relevant for power analysis (e.g., having a CI). Instead, similar power estimates could potentially be obtained using different inferential methods, such as where the joint significance test recommends 74 participants and a bootstrapping method recommends 78 participants for medium effects at 80\% power (Fritz \& MacKinnon, 2007).

To explore current practices in power analysis for mediation, we conducted a brief literature review of articles published in Psychological Science in 2020 because of the journal's requirement for a justification of sample size selection. There were 36 articles that included the word 'mediation'. Seven articles were excluded: Four that mentioned mediation as a future direction, a meta-analysis, a commentary, and one that used the word mediation in a different context. The Supplemental Material includes the remaining 29 articles, indicated with a $*$ in the reference list. Only one article did a statistical power analysis for the mediation analysis, using the Monte Carlo CI method for power analysis then the percentile bootstrap CI for data analysis. Twelve articles included a power analysis for an analysis other than the mediation, and 16 articles had no mention of a power analysis at all. More guidance and recommendations for power analysis procedures 
for mediation analysis are needed for documented sample size planning to become a common practice. Power analysis for mediation is so uncommon that we cannot rely on a common practice to understand how it is currently done, so the aim of this paper is to explore a possible way to reduce the computational time required for the process.

We seek to aid researchers in estimating appropriate sample sizes for mediation analysis, including cases where assumptions of normality and homoskedasticity are violated. First we describe how to estimate a mediation model for between-subjects designs with one mediator, and overview six inferential methods testing for mediation. Then, we discuss choosing an inferential method and the implications of that method for power analysis. We give results from a simulation study comparing statistical power for each inferential method for the same sample sizes, including when data violate the statistical assumptions of linear regression. We conclude with recommendations for when to choose a more computationally efficient inferential method for power analyses and when to switch to a more robust, time-intensive inferential method that matches the inferential method used for data analysis.

\section{Inferential Methods for Mediation Analysis}

In a mediation model, a predictor variable $(X)$ affects an outcome variable $(Y)$ through a mediator variable $(M)$. Figure 1 shows the total effect of $X$ on $Y$ as path $c$, without the mediator (top), and the direct effect of $X$ on $Y$ in the mediation represented by $c^{\prime}$ (bottom). The indirect effect $a b$ is how much $Y$ changes as a result of the $X$ variable manipulation ${ }^{1}$ through $M$.

\footnotetext{
${ }^{1} \mathrm{X}$ is a dichotomous variable with equal allocation to represent random assignment to one of two experimental conditions
} 


\section{Figure 1}

\section{Mediation Analysis Path Diagram}

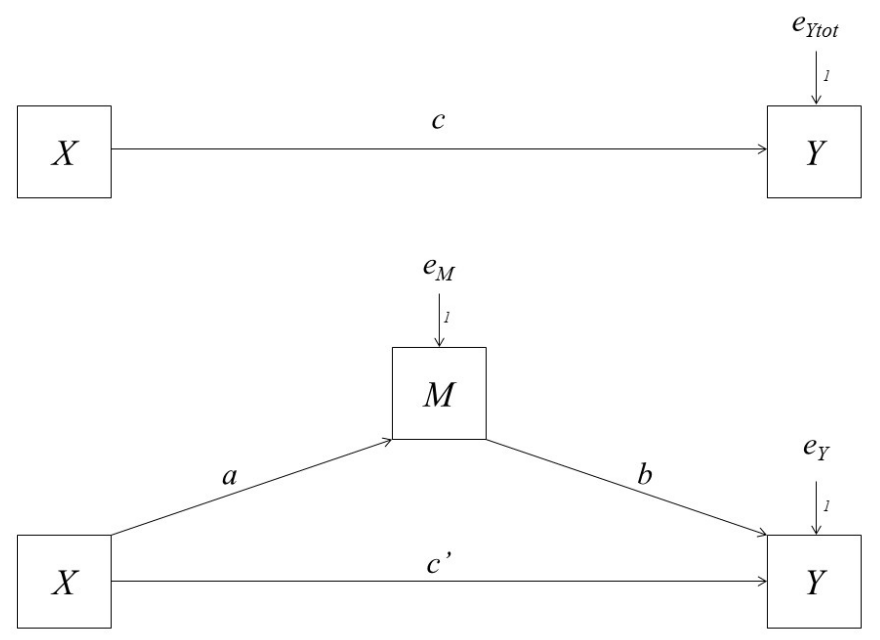

Mediation analysis in this regression framework can be thought of as two regression analyses, which must meet several statistical assumptions (Poole \& O'Farrell, 1971). These assumptions of regression involve the conditional distribution of the dependent variable: linearity, independence, homoskedasticity of variance, and normality (Poole \& O'Farrell, 1971).

This paper focuses on six inferential methods for the indirect effect: the causal steps approach (Baron \& Kenny, 1986), the joint significance test (MacKinnon et al., 2002), the Sobel test (Sobel, 1982), the percentile bootstrap CI (Bollen \& Stine, 1990), the bias-corrected bootstrap CI (Shrout \& Bolger, 2002), and the Monte Carlo CI (MacKinnon et al., 2004). For all the tests, the $\alpha$-level must be pre-determined. Table 1 provides a comparison of these inferential methods in terms of robustness to assumption violations, computational intensity, power, and type 1 error rate based on prior literature.

Causal Steps Approach. The causal steps approach requires four null hypothesis significance tests to conclude a mediated effect exists: 1) the total effect of $X$ on $Y$ (c-path) 2) the effect of $X$ on $M$ (a-path), 3) the effect of $M$ on $Y$ controlling for $X$ (b-path), and 4) the direct effect $\left(c^{\prime}\right)$ to determine partial or complete mediation, though that is not a useful distinction (Hayes, 2009, Rucker et al., 2011). If tests 1-3 are all 
significant, the causal steps approach rejects the null hypothesis that there is no mediation.

Joint Significance Test. The requirement for the total effect to be significant in order to find a significant indirect effect may cause a mediated effect to be overlooked (MacKinnon et al., 2002 Preacher \& Hayes, 2004). For the joint significance test, if both the $a$-path and $b$-path are significant (i.e., steps 2 and 3 above), then the researcher would conclude that mediation is present (Kenny et al., 1998). The joint significance test has a closed-form solution and so direct calculation of power is possible, making it potentially even more computationally efficient (O’Rourke \& MacKinnon, 2014).

Sobel Test. The Sobel test first estimates the indirect effect, then the standard error of the indirect effect using equations given in Sobel $(1982)$. The estimated indirect effect is divided by its standard error estimate, producing a Z-statistic that can be compared to a standard normal distribution to conclude if mediation is present.

Percentile Bootstrap CI. Bootstrapping for mediation resamples with replacement the number of observations from the original sample and an indirect effect is calculated from the resampled data (Bollen \& Stine, 1990). This process is repeated many times to get many estimates of the indirect effect, then the percentile bootstrap CI constructs a CI by ordering these estimates from lowest to highest (Efron \& Tibshirani, 1993). Any values above the $100 \times \frac{\alpha}{2}$ percentile and below the $100 \times\left(1-\frac{\alpha}{2}\right)$ percentile are included in the interval. If zero is not included in the interval, then the researcher can conclude that mediation is present. When analyzing data, the percentile bootstrap CI is the default in the commonly used software package PROCESS (Hayes, 2013).

Bias-corrected Bootstrap CI. Using the same process for generating bootstrap samples, the bias-corrected bootstrap CI method adjusts the cutoffs to account for differences between the median of the bootstrap sampling distribution and the true indirect effect. The cutoffs are calculated by doubling the Z-score corresponding to the proportion of bootstrap estimates less than the original sample estimate and adding it to the cutoffs used for the percentile bootstrap CI (Efron \& Tibshirani, 1993). 
Monte Carlo CI. Monte Carlo CIs use sample estimates to generate estimated sampling distributions of $\hat{a}$ and $\hat{b}$ separately using a normal distribution, then combine them to create an estimated sampling distribution for $\widehat{a b}$ where the $100 \times \frac{\alpha}{2}$ and $100 \times\left(1-\frac{\alpha}{2}\right)$ percentiles form a CI (MacKinnon et al., 2004). If zero is not contained within the interval, then mediation is present.

The distribution of the product confidence method is asymptotically equivalent to the Monte Carlo CI and is more computationally efficient; this procedure is available in the RMediate package (Tofighi \& MacKinnon, 2011), but since it is originally written in closed source software we were unable to implement it in our simulation as one of the comparable methods. 


\section{Table 1}

Comparison of Inferential Methods

Statistical Properties

Robustness to Assumption All methods rely on the assumptions of linearity and in-

Violations (Biesanz et al., dependence. Bootstrapping methods are the only methods 2010) included that do not rely on the assumptions of normality and homoskedasticity of residuals.

Computational Intensity Bootstrapping methods are the most computationally in(Efron \& Tibshirani, 1993 tensive. Monte Carlo CI requires drawing estimates from O'Rourke \& MacKinnon, a theoretical distribution, but not from the sample like the 2014; Shrout \& Bolger, bootstrap. The remaining tests are more computationally 2002; Yzerbyt et al., 2018) efficient, and in particular the joint significance and Sobel tests where power can be simulated very quickly or computed analytically

Power (Fritz \& MacKinnon, The bias-corrected bootstrap CI has been shown in simu2007; Yzerbyt et al., 2018) lation studies to have the highest power, followed by the joint significance test, percentile bootstrap CI, and Monte Carlo CI which are similar to each other.

Type 1 Error Rate (Hayes \& The bias-corrected bootstrap CI has been shown to have Scharkow, 2013 Yzerbyt et inflated type 1 error rate, and the causal steps approach al., 2018) and Sobel test have very conservative type 1 error rates. The joint significance test, percentile bootstrap CI, and Monte Carlo CI all have a similar, appropriate type 1 error rates. 


\section{Power Analysis in Mediation}

Power for between-subjects mediation is determined by the following values: sample size; population effect size as set by path coefficients $a, b$, and $c^{\prime} ; \alpha$-level; and the inferential method used (for discussion of factors to consider for mediation with repeated measures designs, see Montoya, 2022). Estimating statistical power for mediation relies on the product of two regression coefficients which does not follow a normal distribution (Lomnicki, 1967), so power analyses using simulation methods are preferred for the indirect effect due to their ability to generate the nonnormal distribution of the product (Schoemann et al., 2017). Conducting a simulation for power analysis involves generating thousands of samples of data. When an analytical formula is available for power analysis, it is possible to calculate sample size from power, and power from sample size. With simulation methods, it is only possible to calculate power given sample size because thousands of samples of data from that set sample size must be simulated. This means it is often necessary to repeat the whole simulation several times for different sample sizes until a sample size is found that achieves the desired level of power, adding to the computational burden.

\section{Software Packages}

Most software for power analysis for mediation implements only one or two of the discussed inferential methods. The available software packages also assume normality and homoskedasticity. No software package yet exists to output a sample size using a bootstrapping method, though bmem (Zhang \& Wang, 2013) and WebPower (Zhang \& Yuan, 2018) can estimate power using bootstrapping from a given sample size. For tools that can estimate a sample size for a desired power, the $\mathrm{R}$ package pwr2ppl (Aberson, 2019b) uses the joint significance test, the Shiny app MCpowrMed (Schoemann et al., 2017) uses the Monte Carlo CI, and the web application and R package WebPower (Zhang \& Yuan, 2018) use the Sobel test by default. 
Power analyses using different inferential methods differ in their computational ease. In R 4.1.1 on a laptop computer running macOS Big Sur, estimating power with the joint significance test or Sobel test takes less than a second with 1000 repetitions with path coefficients of .26 and a sample size of 200, and the Monte Carlo CI takes only a few seconds under the same conditions drawing 1000 samples from the random normal sampling distribution for each repetition. Power analysis using bootstrapping is much more time consuming because each simulated sample must be bootstrapped thousands of times, taking over 24 hours with 1000 repetitions and 5000 bootstraps ${ }^{2}$. Researchers typically repeat this process for many different possible sample sizes and coefficient estimates for power analyses. For example, if a sample size of 200 does not have sufficient power, they then need to choose a higher sample size and calculate power again. If that sample size, say 250 , gives power that is higher than necessary, they would need to try a smaller sample size between 200 and 250. This iterative process goes on until a sample size is found that yields adequate statistical power. This raises the question: Is bootstrapping, the recommended method for data analysis, worth the additional computational resources for power analysis?

\section{Choosing an Inferential Method for Power Analysis and Data Analysis}

No method is optimal for both power analysis, which values computational efficiency, and data analysis, where the percentile bootstrap CI with its robustness to violations of statistical assumptions and confidence interval production is preferred (Hayes, 2009). The benefit of an inferential method being robust to violations of assumptions of normality and homoskedasticity in power analysis are still relevant, though in this process where each repetition is a dichotomous decision of whether or not the null hypothesis can be rejected a CI is not necessary. If different inferential methods agree in terms of sample size recommendations, then the more computationally efficient method could be used for a

\footnotetext{
2 Future research should examine the sensitivity of power analysis using fewer number of bootstraps than intended for the original data analysis. Using 1000 bootstraps instead of 5000, the time required for this power analysis using the WebPower $\mathrm{R}$ package decreases to around six hours.
} 
power analysis to get close to the appropriate sample size, and then the more robust percentile bootstrap CI could be used for data analysis.

In summary, power analysis for mediation requires researchers to make a choice about which inferential method they want to use. Bootstrapping methods are the preferred method for data analysis, but they are very computationally intensive to the point where few tools are available for power analysis with bootstrapping. Additionally, no tools exist that output a recommended sample size given a desired level of power. Previous research shows that the sample sizes required for $80 \%$ power from the joint significance test, the percentile bootstrap CI, and the Monte Carlo CI are very similar (Fritz \& MacKinnon, 2007). This suggests that the joint significance test could be a reasonable method for approximating a sample size in a power analysis close enough to the percentile bootstrap CI. This needs to be empirically tested. The use of different inferential methods for power analysis and data analysis also has yet to be examined in situations where data do not meet the statistical assumptions of normality and homoskedasticity.

\section{The Present Study}

This research builds on sample size recommendations among various inferential methods from Fritz and MacKinnon (2007). We test if power estimates for the same sample size at a given $\alpha$-level agree enough across inferential methods to use a more computationally-efficient approach than the percentile bootstrap CI for power analysis. As an example, our simulation shows when both $a$ and $b$ are set to .59 , the joint significance test recommends a sample size of 92 to achieve $80 \%$ power. Using a sample size of 92 and the same parameter coefficients, the percentile bootstrap CI achieves a power of $80.7 \%$. If a similar pattern holds across many coefficient estimates, researchers could reasonably use a less computationally intensive method for power analysis. We also discuss the agreement of these methods in power analysis when assumptions of normality and homoskedasticity are violated, and offer suggestions for researchers when they know a priori that their data will 
violate these assumptions.

\section{Disclosures}

\section{Preregistration}

This study was preregistered at https://osf.io/ebq9gs. The preregistered measure of agreement was power estimates within 5 percentage points for the same sample size and effect size combination. We determined agreement between each method and the percentile bootstrap CI. This is a slight deviation from our preregistration, in which all methods were intended to be compared in a pairwise manner to each other. Upon peer review, we determined this to be a more effective test of our question, as it focuses on the percentile bootstrap CI as the proposed data analytic method, and explores all other methods as the method used for power analysis.

\section{Data, materials, and online resources}

Simulation code, results, and data analysis scripts are available at https://osf.io/tnpu7/.

\section{Reporting}

We report all simulations and analyses conducted as part of this work.

\section{Method}

The purpose of this simulation study was to examine how aligned power estimates are among inferential methods for mediation analysis. We do this by comparing six inferential methods for the indirect effect at the same sample sizes, including when data violate the statistical assumptions of linear regression. For each inferential method, 10,000 samples of data were generated at each sample size until the simulation found a sample size with $80 \%$ power (within 0.1 percentage points) at $\alpha=.05$. We then generated 10,000 samples of data with that sample size and did a power analysis using the percentile 
bootstrap CI to see if the estimates agreed. We completed this process for four different parameter values on both the $a$-path and the $b$-path for a total of 16 different combinations of effect sizes. We generated data that met all the statistical assumptions of linear regression, six conditions that violate the normality assumption to varying degrees, and three conditions that violate the homoskedasticity assumption. The primary outcome was the proportion of times the percentile bootstrap CI had power within in the range of 75\%-85\% using the sample size required to achieve $80 \%$ power for each other inferential method. The Supplemental Material also explores this process for negative parameter values and power higher than $80 \%$.

\section{Data Generation}

We used GAUSS 19 on a Windows server to generate data for three variables: $X, M$, and $Y$, where $X$ is 0 or 1 with equal allocation to represent random assignment to one of two experimental conditions. Small (.02), medium (.15), and large (.35) $R^{2}$ effects were used (Cohen, 1988), along with an effect between small and medium (.07) that is commonly observed in psychological research (Fritz \& MacKinnon, 2007). Path coefficients on the $a$-path and the $b$-path were calculated correspondingly by taking the square root of these $R^{2}$ effect sizes, resulting in the following four path coefficients: .14, .26, .39, and .59. $M$ was generated with path coefficients $a$ and $e_{M}$ was randomly generated from a standard normal distribution. $Y$ was generated using path coefficients $b, c^{\prime}$ was always .39 because it only affects the causal steps approach (Baron \& Kenny, 1986, Fritz \& MacKinnon, 2007), and $e_{Y}$ was randomly generated from a standard normal distribution. The intercepts were always set to 0 . The equations used for data generation are available in the Supplemental Material.

Data were also generated with varying levels of nonnormality and heteroskedasticity of residuals using the same effect size combinations. Six variations of nonnormal data were generated using the procedure from Fleishman (1978) to account for slight or extreme skew 
of the residuals of just the $M$ variable, just the $Y$ variable, or both. Three conditions of heteroskedastic residuals were generated using the procedure from Long and Ervin (2000): $M, Y$, and both $M$ and $Y$. Full details of the data generating procedures for assumption violations are available in the Supplemental Material.

\section{Data Analysis and Inferential Method Agreement}

Power was calculated as the proportion of samples that detected a significant indirect effect out of the 10,000 samples. For bootstrapping methods, each sample was bootstrapped 5,000 times. For the Monte Carlo CI, 10,000 estimates were drawn from the sampling distribution for each CI. Each inferential method was used to find the sample size at which it had $80 \%$ power for each effect size combination. That sample size was then used in a power analysis with the percentile bootstrap confidence interval to estimate its power at the same sample size as the other inferential method. For example, we first used the Sobel test with parameters $a=.14$ and $b=.14$ for data that met the regression assumptions, iterating through sample sizes until we found it needs a sample size of 1749 for $80 \%$ power. We then used the percentile bootstrap CI with a sample size of 1749 to calculate power, which was $83 \%$. Each inferential method was considered in agreement with the percentile bootstrap CI for power analysis if, for that sample size in each condition, the percentile bootstrap CI had statistical power between $75 \%-85 \%$ using the percentile bootstrap CI.

\section{Results}

Table 2 includes the proportion of times the percentile bootstrap CI achieved power between $75 \%-85 \%$ using the sample size determined from each of the inferential methods for the same effect size combination. The columns represent the assumption violation conditions, where either no regression assumptions were violated, normality was violated, or homoskedasticity was violated. For example, when the sample size required for $80 \%$ power with the Sobel test gives a power estimate of $83 \%$ with the percentile bootstrap CI 
for the same parameter combination, those inferential methods would be in agreement. This overall proportion of agreement is in Table 2, where the third row is the Sobel test and the second column is the proportion of agreement with no assumption violations. Thus, .69 represents the proportion of path coefficients for which the percentile bootstrap confidence interval found power between 75\%-85\% when using the sample size required for 80\% power from the Sobel test assuming normal and homoskedastic residuals. 


\section{Table 2}

Agreement Proportion with the Percentile Bootstrap CI

Assumption Violation Condition

\begin{tabular}{llll}
\hline $\begin{array}{l}\text { Inferential Method for Power } \\
\text { Analysis }\end{array}$ & $\begin{array}{l}\text { No Assumption } \\
\text { Violations }\end{array}$ & $\begin{array}{l}\text { Nonnormal } \\
\text { Residuals }\end{array}$ & $\begin{array}{l}\text { Heteroskedastic } \\
\text { Residuals }\end{array}$ \\
\hline Causal Steps Approach & .81 & .81 & .81 \\
Joint Significance Test & 1.00 & 1.00 & .79 \\
Sobel Test & .69 & .68 & .83 \\
Bias-corrected Bootstrap CI & .69 & .69 & .71 \\
Monte Carlo CI & 1.00 & 1.00 & .79 \\
\hline
\end{tabular}

Note. Proportion of times the sample size produced by each inferential method in a power analysis also had between $75 \%-85 \%$ power using the percentile bootstrap CI for data analysis, split by assumption violation conditions.

Two methods had agreement in all conditions with the percentile bootstrap confidence interval when the assumptions of linear regression were met: the joint significance test and the Monte Carlo CI. For all 16 parameter combinations included in this simulation, these two inferential methods produced sample sizes for $80 \%$ power that had power between $75 \%-85 \%$ for the percentile bootstrap CI. Using sample sizes from the joint significance test, power estimates for the percentile bootstrap CI ranged from 79.0\%-82.2\%, and sample sizes from the Monte Carlo CI had power estimates for the percentile bootstrap CI ranging from 79.0\%-81.1\%. The largest observed difference among the inferential methods that met that criteria was $2.1 \%$ off using the joint significance test. The sample sizes required for $80 \%$ power using the causal steps approach, the Sobel test, and the bias-corrected bootstrap CI did not always agree with the percentile bootstrap CI. However, this disagreement only occurred for certain parameter combinations; see Table S2 
in the Supplemental Material for details.

The causal steps approach, Sobel test, and bias-corrected bootstrap CI had low agreement with the percentile bootstrap CI across all conditions, with power as low as $72 \%$ using the sample size from the bias-corrected bootstrap CI in a power analysis using the percentile bootstrap CI when no assumptions were violated. Inferential methods that are not considered to agree with to the percentile bootstrap CI in this simulation tended to estimate consistently higher sample sizes (causal steps approach and Sobel test, where the sample size requred for $80 \%$ power reached $89.8 \%$ power using the percentile bootstrap CI) or lower sample sizes than the percentile bootstrap CI (bias-corrected bootstrap CI). Table 3 gives the ranges of power the percentile bootstrap CI achieved using sample sizes from each of the six inferential methods. When assumptions are violated, the differences in power estimated using the percentile bootstrap CI compared to either the causal steps approach or the Sobel test differ even more drastically. 


\section{Table 3}

Ranges of Power using the Percentile Bootstrap CI

\begin{tabular}{llll}
\hline $\begin{array}{l}\text { Inferential Method for Power } \\
\text { Analysis }\end{array}$ & $\begin{array}{l}\text { No Assumption } \\
\text { Violations }\end{array}$ & $\begin{array}{l}\text { Nonnormal } \\
\text { Residuals }\end{array}$ & $\begin{array}{l}\text { Heteroskedastic } \\
\text { Residuals }\end{array}$ \\
\hline Causal Steps Approach & $79.2-88.4$ & $77.8-89.2$ & $63.5-85.7$ \\
Joint Significance Test & $\mathbf{7 9 . 1 - 8 0 . 9}$ & $\mathbf{7 8 . 5 - 8 3 . 1}$ & $62.8-81.2$ \\
Sobel Test & $79.8-89.8$ & $79.3-90.8$ & $66.1-89.5$ \\
Percentile Bootstrap CI & $\mathbf{7 9 . 3 - 8 0 . 9}$ & $\mathbf{7 9 . 3 - 8 1 . 8}$ & $\mathbf{7 8 . 6 - 8 1 . 6}$ \\
Bias-corrected Bootstrap CI & $72.6-80.6$ & $71.2-80.6$ & $71.8-80.2$ \\
Monte Carlo CI & $\mathbf{7 9 . 6 - 8 1 . 1}$ & $\mathbf{7 8 . 6 - 8 3 . 3}$ & $62.1-81.6$ \\
\hline
\end{tabular}

Note. Range of powers achieved between inferential methods (\%), with bold indicating the inferential methods had agreement across all coefficient estimates. All estimates are power from the percentile bootstrap CI using sample sizes required to achieve $80 \%$ power with each inferential method in the first column.

\section{Assumption Violations}

When the normality assumption is violated, the joint significance test and the Monte Carlo CI agree with the percentile bootstrap CI, and in these conditions the largest difference in power among agreeing methods was 3.64\%. When the homoskedasticity assumption is violated, the pattern of agreement does not always hold. The sample sizes recommended by the joint significance test and the Monte Carlo CI each only have power between $75 \%-85 \%$ using the percentile bootstrap confidence interval in .79 of the conditions with heteroskedastic residuals. When just the residuals of $M$ are heteroskedastic, power ranges from $78.5 \%-81.2 \%$ with joint significance test sample sizes and power ranges from 79.1\%-81.6\% with the Monte Carlo CI. The disagreement occurs in cases where the 
residuals of the $Y$ variable are heteroskedastic. In those cases, the power for the percentile bootstrap CI using estimates from the joint significance test or Monte Carlo CI could be as low as $63 \%$ or $62 \%$, respectively. The most extreme values occurred at the highest degree of nonnormality for both $M$ and $Y$ residuals.

\section{Discussion}

When performing a power analysis for a mediation analysis, researchers have a variety of inferential methods to choose from. For data analysis previous research suggests the percentile bootstrap confidence interval performs best, but is perhaps too computationally intensive to be used for power analysis. Sample size recommendations from five of the most commonly used inferential methods for the indirect effect were compared to the percentile bootstrap confidence interval with respect to achieved power. Under the assumptions of linear regression, the joint significance test and the Monte Carlo CI had power estimates that agreed with the percentile bootstrap CI for the same sample sizes? This fits with the previous findings that these inferential methods have similar trade-offs between power and type 1 error rates (Hayes \& Scharkow, 2013, MacKinnon et al., 2002). The joint significance test is the least computationally intensive inferential method of the three and also includes a closed-form solution for power (O'Rourke \& MacKinnon, 2014). Tools such as the R package pwr2ppl (Aberson, 2019a) can be used to do power analysis with the joint significance test with an a priori expectation of meeting normality and homoskedasticity assumptions.

When the a priori expectation is that the normality or homogeneity assumptions are violated, more caution must be used when starting a power analysis. When the assumption of normality is violated, the joint significance test and Monte Carlo CI agree with the percentile bootstrap CI in a power analysis. This is the case when just the $M$ variable is

\footnotetext{
${ }^{3}$ Due to the similarity in performance between the Monte Carlo CI and the distribution of the product method, any claims about the Monte Carlo CI can also be applied to the distribution of the product method.
} 
nonnormal, just the $Y$ variable is nonnormal, and both the $M$ and the $Y$ variable is nonnormal, at least to the degree that the normality assumption was violated in these simulation conditions. When the assumption of heteroskedasticity is expected to be violated in the $Y$ residuals, the joint significance test and the Monte Carlo CI do not always agree with the percentile bootstrap CI. Here, the percentile bootstrap CI requires larger sample sizes than the joint significance test and the Monte Carlo CI. The joint significance test, percentile bootstrap CI, and Monte Carlo CI all agree regardless of the $M$ residuals being heteroskedastic or at least to the degree that the homoskedasticity assumption was violated in these simulation conditions 4 .

While the current research suggests that under some conditions the joint significance test can be used for sample size selection when the percentile bootstrap CI will be used for data analysis, these findings are limited by the effect sizes and other data generating conditions used in this study. One approach researchers could take is using the joint significance test to hone in on a sample size, then check the power of that sample size using a power analysis with percentile bootstrap CI. This would require only one run of the percentile bootstrap CI (which will admittedly take a while), but can help to ensure that the results from the joint significance test are valid. If the percentile bootstrap CI does not return a power close to the desired power, the researcher may want to continue to iterate the sample size using the percentile bootstrap CI. These features of the joint significance test can be beneficial and time saving in the beginning stages of a power analysis where many different sample sizes may be tested. The goal is to get a more strategic starting value for doing the final power analysis using the more time consuming percentile bootstrap CI. The causal steps approach, Sobel test, and bias-corrected bootstrap CI do not produce similar enough estimates to be recommended for use in a power analysis if the percentile bootstrap CI will be used for data analysis, and researchers should be careful to

\footnotetext{
4 The heteroskedastic residuals for $M$ and $Y$ were generated differently due to $X$ being a dichotomous predictor and $M$ being a continuous predictor of just $Y$. Additional details are included in the Supplementary Material
} 
not use power analysis tools that rely on these inferential methods if they intend to use the percentile bootstrap CI for data analysis.

Even in cases where the joint significance test and the Monte Carlo CI have agreement in terms of power with the percentile bootstrap CI when there are assumption violations, the required sample sizes can be very different from when these assumptions are met. For example, with medium parameter coefficients (both the $a$-path and $b$-path are .39 ), the joint significance test requires a sample size of 210 with homoskedastic residuals, but when $M$ has heteroskedastic residuals the required sample size is 340. Researchers should take that into account and simulate data that includes the assumption violations they expect a priori for the most accurate power analysis.

\section{Limitations and Future Directions}

This study considered inferential methods to agree if power was within a 5 percentage point margin of error. Due to the arbitrary nature of the cut-off decided in preregistration, even the inferential methods that were considered in agreement were not exactly the same. One implication of this is the recommendation to start with the joint significance test in a power analysis could produce starting sample sizes with varying degrees of accuracy.

Another limitation is that all the information about handling violations of statistical assumptions requires the researcher to simulate data for their power analyses that violate the expected assumptions. Additional tools need to be developed for power analysis in mediation that allows expected degree of assumption violations as an input. Based on the simulation results, caution should be used in particular when selecting a sample size when the heteroskedasticity assumption will be violated.

Additional research can be done to generalize these findings to other analyses beyond mediation analysis, such as moderated mediation which involves interactions in a mediation analysis. Research on power analysis is needed in this area as more and more 
studies are investigating interactions in mediation analyses. Larger sample sizes are generally necessary to detect interactions (Aguinis \& Stone-Romero, 1997), so power analyses to determine appropriate sample sizes are necessary. Multilevel modeling using mediation models is also possible (Preacher et al., 2010), and these models are much more complex and computationally intensive to fit. Future research on power analysis in multilevel mediation models could aid researchers in estimating sample sizes for these models, adding to the only tool available for power analysis in multilevel mediation, PowerUpR (Kelcey et al., 2021). Similarly, maximum likelihood estimation is common in structural equation modeling used for mediation analysis, and is another iterative, computationally intensive process which makes power analysis difficult. Similar to this research, it may be possible to use OLS to more efficiently approximate required sample size for maximum likelihood estimation.

\section{Summary}

When assumptions of linear regression are met, sample size required for $80 \%$ power using the joint significance test and the Monte Carlo CI agrees with the percentile bootstrap CI. This pattern tends to hold when the assumption of normality is violated, but not homoskedasticity. When researchers want to use the percentile bootstrap CI for data analysis to get more robust mediation results with a CI, a good starting sample size for a power analysis can be more quickly found using the less computationally intensive joint significance test in the iterative process of inputting sample sizes to calculate power. Prioritizing an inferential method that is quick to compute for power analysis will save time, then the percentile bootstrap CI can verify and ultimately determine the sample size. The percentile bootstrap CI should also be used for data analysis because it is more robust to violations of statistical assumptions and also gives a confidence interval. 


\section{Author Contributions}

A. K. Montoya generated the idea for the study. J. L. Fossum programmed the simulation study and analyzed the data, under the guidance of A. K. Montoya. J. L. Fossum wrote the first draft of the manuscript, and both authors critically edited it with

revisions suggested by A. K. Montoya. Both authors approved the final submitted version of the manuscript.

\section{Conflicts of Interest}

The authors declare that there were no conflicts of interest with respect to the authorship or the publication of this article.

\section{Acknowledgements}

Special thanks to Dr. Katherine Karlsgodt and the QRClab for providing feedback on this manuscript.

\section{Funding}

This material is based upon work supported by the Graduate Research Mentorship award through the Graduate Division of the University of California, Los Angeles awarded to J. L. Fossum and work supported by the UCLA Equity, Diversity, and Inclusion Faculty Career Development Award awarded to Dr. Montoya.

\section{Supplemental Material}

Supplemental material is available at https://osf.io/tnpu7/

\section{Prior Versions}

A preprint of this manuscript is available on PsyArXiv:

https://psyarxiv.com/5tm $2 \mathrm{x}$ 


\section{References}

Aberson, C. L. (2019a). Applied power analysis for the behavioral sciences (2nd). Routledge.

Aberson, C. L. (2019b). Pwr2ppl:Power analysis for common designs. R package version 0.1. https://cran.r-project.org/web/packages/pwr2ppl/index.html

Aguinis, H., \& Stone-Romero, E. F. (1997). Methodological artifacts in moderated multiple regression and their effects on statistical power. Journal of Applied Psychology, 82, $192-206$.

Anderson, S. F., Kelley, K., \& Maxwell, S. E. (2017). Sample-size planning for more accurate statistical power: A method adjusting sample effect sizes for publication bias and uncertainty. Psychological Science, 28, 1547-1562.

Baron, R. M., \& Kenny, D. A. (1986). The moderator--mediator variable distinction in social psychological research: Conceptual, strategic, and statistical considerations. Journal of Personality and Social Psychology, 51(6), 1173-1182.

Biesanz, J. C., Falk, C. F., \& Savalei, V. (2010). Assessing mediational models: Testing and interval estimation for indirect effects. Multivariate Behavioral Research, 45, $661-701$.

Bollen, K. A., \& Stine, R. (1990). Direct and indirect effects: Classical and bootstrap estimates of variability. Sociological Methodology, 20, 115-140.

Cohen, J. (1988). Statistical power analysis for the behavioral sciences (2nd). NJ: Lawrence Erlbaum Associates.

Cohen, J. (1992). A power primer. Psychological Bulletin, 112, 155-159. https://doi.org/https://doi.org/10.1037/0033-2909.112.1.155

Efron, B., \& Tibshirani, R. J. (1993). An introduction to the bootstrap. Chapman \& Hall.

Fleishman, A. I. (1978). A method for simulating non-normal distributions. Psychometrika, $43(4)$.

Fritz, M. S., \& MacKinnon, D. P. (2007). Required sample size to detect the mediated effect. Psychological Science, 18(3), 233-239. 
Hayes, A. F. (2009). Beyond Baron and Kenny: Statistical mediation analysis in the new millennium. Communication Monographs, 76(4), 408-420.

Hayes, A. F. (2013). Introduction to mediation, moderation, and conditional process analysis. Guilford Press.

Hayes, A. F., \& Scharkow, M. (2013). The relative trustworthiness of inferential tests of the indirect effect in statistical mediation analysis: Does method really matter? Psychological Science, 24, 1918-1927.

Kelcey, B., Xie, Y., Spybrook, J., \& Dong, N. (2021). Power and sample size determination for multilevel mediation in three-level cluster-randomized trials. Multivariate Behavioral Research, 56(3), 496-513. https://doi.org/10.1080/00273171.2020.1738910

Kenny, D. A., Kashy, D. A., \& Bolger, N. (1998). Data analysis in social psychology. In D. T. Gilbert, S. T. Fiske, \& G. Lindzey (Eds.), The handbook of social psychology (pp. 233-265). McGraw-Hill.

Lomnicki, Z. A. (1967). On the distribution of products of random variables. Journal of the Royal Statistical Society, 29, 513-524.

Long, J. S., \& Ervin, L. H. (2000). Using heteroskedasticity consistent standard errors in the linear regression model. American Statistician, 54(3), 217-224. https://doi.org/10.2307/2685594

MacKinnon, D. P., Lockwood, C. M., Hoffman, J. M., West, S. G., \& Sheets, V. (2002). A comparison of methods to test mediation and other intervening variable effects. Psychological Methods, 7(1), 83-104.

MacKinnon, D. P., Lockwood, C. M., \& Williams, J. (2004). Confidence limits for the indirect effect: Distribution of the product and resampling methods. Multivariate Behavioral Research, 39, 99-128. 
Montoya, A. K. (2022). Selecting a within- or between-subject design for mediation: Validity, causality, and statistical power. Multivariate Behavior Research. https://doi.org/10.1080/00273171.2022.2077287

O'Rourke, H. P., \& MacKinnon, D. P. (2014). When the test of mediation is more powerful than the test of the total effect. Behavior Research Methods, 47, 424-442.

Poole, M. A., \& O'Farrell, P. N. (1971). The assumptions of the linear regression model. Transactions of the Institute of British Geographers, 52, 145-158.

Preacher, K. J., \& Hayes, A. F. (2004). Spss and sas procedures for estimating indirect effects in simple mediation models. Behavior Research Methods, 36, 717-731.

Preacher, K. J., Zyphur, M. J., \& Zhang, Z. (2010). A general multilevel SEM framework for assessing multilevel mediation. Psychological Methods, 15(3), 209-233. https://doi.org/10.1037/a0020141

Rucker, D. D., Preacher, K. J., Tormala, Z. L., \& Petty, R. E. (2011). Mediation analysis in social psychology: Current practices and new recommendations. Social and Personality Psychology Compass, 5, 359-371.

Schoemann, A. M., Boulton, A. J., \& Short, S. D. (2017). Determining power and sample size for simple and complex mediation models. Social Psychological and Personality Science, 8(4), 379-386. https://doi.org/10.1177/1948550617715068

Shrout, P. E., \& Bolger, N. (2002). Mediation in experimental and nonexperimental studies: New procedures and recommendations. Psychological Methods, 7, 422-445.

Sobel, M. E. (1982). Asymptotic confidence intervals for indirect effects in structural equation models. Sociological Methodology, 13, 290-312.

Tofighi, D., \& Mackinnon, D. P. (2011). Rmediation: A R package for mediation analysis confidence intervals. Behavior Research Methods, 43, 692-700. https://doi.org/10.3758/s13428-011-0076-x

Yzerbyt, V., Muller, D., Batailler, C., \& Judd, C. M. (2018). New recommendations for testing indirect effects in mediational models: The need to report and test 
component paths. Journal of Personality and Social Psychology: Attitudes and

Social Cognition, 115(6), 929-943. https://doi.org/10.1037/pspa0000132

Zhang, Z., \& Wang, L. (2013). Methods for mediation analysis with missing data. Psychometrika, 78(1), 154-184.

Zhang, Z., \& Yuan, K. H. (2018). Practical statistical power analysis using webpower and $R$. ISDSA Press. 